\title{
Some results on Wiener index of a graph: an overview
}

\author{
Martin Knor, Snježana Majstorović† $\quad$ Riste Škrekovski ${ }^{\ddagger}$
}

\begin{abstract}
The Wiener index $W(G)$ of a connected graph $G$ is defined as the sum of distances between all pairs of vertices in $G$. In 1991, Šoltés [9] posed the problem of finding all graphs $G$ such that equality $W(G)=W(G-v)$ holds for all vertices $v$ in $G$. The only known graph with this property is the cycle $C_{11}$. Our main object of study is the relaxed version of this problem: find graphs for which Wiener index does not change when a particular vertex $v$ is removed. This overview contains results which were obtained and published during the past two years concerning relaxed Šoltés's problem.
\end{abstract}

Keywords: Wiener index, transmission, Cartesian product, induced subgraph

MSC: 05C12, 05C90

\section{Introduction}

Average distance is one of the three most robust measures of network topology, along with its clustering coefficient and its degree distribution. Nowadays it has been frequently used in sociometry and the theory of social networks [4]. Wiener index, defined as the sum of distances between all (unordered) pairs of vertices in a graph, besides its crucial role in the calculation of average distance, is the most famous topological index in mathematical chemistry. It is named after Wiener [10], who introduced it in 1947 for the purpose of determining boiling points of alkanes. Since

\footnotetext{
${ }^{*}$ Slovak University of Technology in Bratislava, Faculty of Civil Engineering, Department of Mathematics, Bratislava, Slovakia. E-Mail: knor@math.sk

${ }^{\dagger}$ Department of Mathematics, Josip Juraj Strossmayer University of Osijek, Osijek, Croatia. E-Mail: smajstor@mathos.hr

${ }^{\ddagger}$ FMF, University of Ljubljana \& Faculty of Information Studies, Novo Mesto \& FAMNIT, University of Primorska, Slovenia. E-Mail: skrekovski@gmail.com
}

DOI: https://doi.org/10.5592/CO/CCD.2018.04 
then Wiener index has become one of the most frequently used topological indices in chemistry, since molecules are usually modeled by undirected graphs. Other applications of this graph invariant can be found in crystallography, communication theory and facility location. Wiener index has also been studied in pure mathematics under various names: the gross status, the distance of a graph, the transmission of a graph etc. It seems that the first mathematical paper on Wiener index was published in 1976 [3]. Since then, a lot of mathematicians have studied this quantity very extensively. A great deal of knowledge on Wiener index is accumulated in survey papers $[2,5,11]$. Nowadays it has been frequently used in sociometry and the theory of social networks [4].

Throughout this paper all graphs will be finite, simple and undirected.

The Wiener index $W(G)$ of a connected graph $G$ is defined as the sum of distances between all (unordered) pairs of vertices in $G$ :

$$
W(G)=\sum_{\{u, v\} \subseteq V(G)} d_{G}(u, v)=\frac{1}{2} \sum_{v \in V(G)} t_{G}(v),
$$

where the distance $d_{G}(u, v)$ between vertices $u$ and $v$ is defined as the number of edges on a shortest path connecting these vertices in $G$, and the distance, or transmission, $t_{G}(v)$ of a vertex $v \in V(G)$ is the sum of distances between $v$ and all other vertices of $G$.

In 1991, Šoltés [9] posed the following problem:

Problem 1. Find all such graphs $G$ that the equality $W(G)=W(G-v)$ holds for all their vertices $v$.

Till now, only one such graph is known: it is a cycle with 11 vertices.

Motivated by Šoltés's problem, in [6] we constructed an infinite family of unicyclic graphs which preserve Wiener index after removal of a particular vertex. In fact, we proved that there are infinitely many unicyclic graphs with this property even when we fix the length of the cycle. Then we showed that for every graph $G$ there are infinitely many graphs $H$ such that $G$ is an induced subgraph of $H$ and $W(H)=W(H-v)$ for some vertex $v \in V(H) \backslash V(G)$. Our research is further extended to graphs in which vertex $v$ is of arbitrary degree, see [7]. For $k \geq 3$ we showed that there are infinitely many graphs $G$ with a vertex $v$ of degree $k$ for which $W(G)=W(G-v)$. Moreover, we proved the existence of such graphs when the degree is $n-1$ or $n-2$. Finally, we showed that dense graphs cannot be a solution of Problem 1. 
Our contribution shows that the class of graphs, for which Wiener index does not change when a particular vertex is removed, is rich. This gives hope that Šoltes's problem may have another solution besides $C_{11}$.

\section{Preliminaries}

Let $G$ be a connected graph. By $d_{G}(v)$ we denote the degree of vertex $v$. A pendent vertex is a vertex of degree one and a pendent edge is an edge incident with a pendent vertex. For a given vertex $v$ of a graph $G$, the eccentricity of $v$, denoted by ecc $(v)$ is defined to be the greatest distance from $v$ to any other vertex in $G$. A diameter $\operatorname{diam}(G)$ of a graph $G$ is the value of the greatest eccentricity in $G$.

By $K_{n}$ we denote an $n$-vertex complete graph and by $S_{n}$ an $n$-vertex star. For more definitions and terminologies in graph theory, see [1]. For Wiener index of the path $P_{n}$ and cycle $C_{n}$ we have very simple formulae. Wiener index of path $P_{n}$ is

$$
W\left(P_{n}\right)=\left(\begin{array}{c}
n+1 \\
3
\end{array}\right)
$$

and Wiener index of a cycle $C_{n}$ is

$$
W\left(C_{n}\right)= \begin{cases}\frac{n^{3}}{8} & \text { if } n \text { is even } \\ \frac{n\left(n^{2}-1\right)}{8} & \text { if } n \text { is odd. }\end{cases}
$$

Proposition 2. Let $G$ be a connected graph and $v \in V(G)$ be a pendent vertex. Let $u v$ be the corresponding pendent edge in $G$ and $G^{\prime}=G-v$. Then

$$
W(G)=W\left(G^{\prime}\right)+t_{G^{\prime}}(u)+n\left(G^{\prime}\right),
$$

where $n\left(G^{\prime}\right)$ is the number of vertices in a graph $G^{\prime}$.

The next statement was proved in [8].

Theorem 3. Let $G_{u}$ and $G_{v}$ be two graphs with $n_{u}$ and $n_{v}$ vertices, respectively, and let $u \in V\left(G_{u}\right), v \in V\left(G_{v}\right)$.

(a) If $G$ arises from $G_{u}$ and $G_{v}$ by connecting $u$ and $v$ by an edge, then

$$
W(G)=W\left(G_{u}\right)+W\left(G_{v}\right)+n_{u} t_{G_{v}}(v)+n_{v} t_{G_{u}}(u)+n_{u} n_{v} .
$$

(b) If $G$ arises from $G_{u}$ and $G_{v}$ by identifying $u$ and $v$, then

$$
W(G)=W\left(G_{u}\right)+W\left(G_{v}\right)+\left(n_{u}-1\right) t_{G_{v}}(v)+\left(n_{v}-1\right) t_{G_{u}}(u) .
$$




\section{Results for unicyclic graphs}

Theorem 4. [6] Let $c \geq 5$. There exists infinitely many unicyclic graphs $G$ with a cycle of length $c$ for which equality $W(G)=W(G-v)$ holds for some $v \in V(G)$.

Proof. Our construction of unicyclic graphs $G$ for which $W(G)=W(G-v)$ goes in the following way. Let $C_{c}$ be a cycle of length $c$. We denote its vertices consecutively by $v_{0}, v_{1}, \ldots, v_{c-1}$. We add to $C_{c}$ a pendent vertex, to obtain a new graph, then we add another pendent vertex (which may be connected to previously added vertex) and so on, until we get a unicyclic graph $G$ with $W(G)=W\left(G-v_{0}\right)$. Then we continue with adding pendent vertices to create infinitely many graphs $G$ with the property $W(G)=W\left(G-v_{0}\right)$. Since $G-v_{0}$ has to be connected, we cannot add pendent vertices to $v_{0}$.

Most of our graphs are obtained from $C_{c}$ by adding a path to $v_{c-1}$ and a tree to $v_{1}$, that is, usually the vertices $v_{2}, v_{3}, \ldots, v_{c-2}$ will all have degree 2 in $G$. By studying the case when $c \in\{3,4\}$, we conclude that there is no unicyclic graph $G$ with a cycle of length $c$ satisfying $W(G)=W(G-v)$ for some $v \in V(G)$. Justification for this conclusion lies in the fact that $W(G)=W(G-v)$ if and only if the following equality holds

$$
t_{G}(v)=\sum_{\left\{u_{1}, u_{2}\right\} \subseteq(V(G) \backslash\{v\})}\left[d_{G-v}\left(u_{1}, u_{2}\right)-d_{G}\left(u_{1}, u_{2}\right)\right] .
$$

If $c=3,4$, then removal of any vertex of degree two from $C_{c}$ will not increase distance between any pair of vertices in $G-v$.

Additionally, we showed that a unicyclic graph $G$ on $n$ vertices for which $W(G)=$ $W(G-v)$ exists if and only if $n \geq 9$.

\section{Induced subgraphs}

By using cycles of certain length, we showed that any graph (even a disconnected one) can be an induced subgraph of some graph $H$ for which $W(H)=W(H-v)$. For this result we needed the following two lemmas.

Lemma 5. [6] Let $C_{c}$ be a cycle of even length, $c=2 a$, such that a is a square. Moreover, let $G_{m}$ be a graph with a vertex $u$ for which $t_{G_{m}}(u)=\frac{a}{3}\left[a^{2}-6 a+2\right]$. Let $H$ be obtained from $G_{m}$ and $C_{c}$ by identifying $u$ with $v_{i}$, where $i=a-\sqrt{a}$. Then $W(H)-W\left(H-v_{0}\right)=0$. 
Lemma 6. [6] Let $C_{c}$ be a cycle of odd length, $c=2 a+1$, such that $4 a+1$ is a square. Moreover, let $G_{m}$ be a graph with a vertex $u$ for which $t_{G_{m}}(u)=\frac{a}{6}\left[2 a^{2}-9 a-5\right]$. Let $H$ be obtained from $G_{m}$ and $C_{c}$ by identifying $u$ with $v_{i}$, where $i=\frac{1}{2}(2 a+1-\sqrt{4 a+1})$. Then $W(H)-W\left(H-v_{0}\right)=0$.

Now using Lemmas 5 and 6 we obtain the following result in which $G$ does not need to be connected.

As a main tool we used Theorem $3 \mathrm{~b}$ in which one graph contains $G$ as an induced subgraph and the other one is a cycle.

Theorem 7. [6] Let $G$ be an arbitrary graph. Then there are infinitely many connected graphs $H$, containing $G$ as an induced subgraph, and such that $W(H)=$ $W\left(H-v_{0}\right)$ for some vertex $v_{0} \in V(H)-V(G)$.

\section{Vertex of a fixed degree}

Our first observation is that if a vertex $v$ has degree 1 in $G$, then $W(G)>W(G-v)$. Since case $d_{G}(v)=2$ was already studied through unicyclic graphs, we focus on $v$ such that $d_{G}(v) \geq 3$. Our main result is the following theorem.

Theorem 8. [7] For every $k \geq 3$ there exist infinitely many graphs $G$ with vertex $v$ such that $d_{G}(v)=k$ and $W(G)=W(G-v)$.

Proof. In each case we show the existence of a graph $G_{1}$ with a vertex $v$ such that $d_{G_{1}}(v)=k$ and $W\left(G_{1}\right)=W\left(G_{1}-v\right)$. Then we construct an infinite class of graphs by attaching to $G_{1}$ a new graph $G_{2}$ according to Theorem 3, and by taking into a consideration necessary and sufficient condition given by (4) under which the resulting graph $H$ satisfies $W(H)=W(H-v)$.

If we consider graphs with $n \geq 7$ vertices, then we can study the case when a degree of $v$ is close to $n$, that is $d(v)=n-1$ or $d(v)=n-2$. In this case we can show the existence of at least one graph $G$ such that $W(G)=W(G-v)$. This is stated in the following theorem.

Theorem 9. [7] Let $n \geq 7$. There exists an $n$-vertex graph $G$ with vertex $v$ so that $d_{G}(v)=n-2$ and $W(G)=W(G-v)$.

Proof. Let $d(v)=n-2$. Cases $n=7,8,9,10$ are considered separately, see [7]. For $n \geq 11$ we take two stars $S_{3}$ and $S_{n-4}$ and connect their central vertices with an 
edge. We add edges between one pendent vertex of $S_{3}$ and $n-10$ pendent vertices of $S_{n-4}$ and denote the resulting graph by $G_{0}$. We take a new vertex $v$ and connect it with all vertices of $G_{0}$ except the central vertex of $S_{3}$. In the resulting graph $G$ we have $d_{G}(v)=n-2, t_{G}(v)=n$ and $\operatorname{diam}(G)=2$. From (4) it follows that $W(G)=W\left(G_{0}\right)$.

Let us now consider the case $d(v)=n-1$. Let $H$ be a graph having $n-1$ vertices and $m$ edges and let $G$ be obtained from $H$ by adding a new vertex $v$ and connecting it by an edge to all vertices of $H$. Then $\operatorname{diam}(G)=2$ and we have

$$
\begin{aligned}
W(G) & =t_{G}(v)+\sum_{\{u, w\} \subseteq V(H)} d_{G}(u, w) \\
& =n-1+2\left(\begin{array}{c}
n-1 \\
2
\end{array}\right)-m \\
& =(n-1)^{2}-m .
\end{aligned}
$$

Since $H=G-v$, we conclude that $W(G)=W(H)$ if and only if

$$
W(H)=(n-1)^{2}-m .
$$

By a computer we checked that for $n-1 \leq 5$ there are no graphs on $n-1$ vertices satisfying (5). Hence we assume that $n-1 \geq 6$. By using (5), for each $n-1 \geq 6$ we managed to construct a graph $H$ on $n-1$ vertices satisfying $W(G)=W(H)$.

\subsection{Graphs with large minimum degree}

At last, we prove that dense graphs cannot be particular solutions of Problem 1. By dense graphs we mean those $n$-vertex graphs in which the minimum degree $\delta(G)$ is at least $n / 2$. Our result relies on the observation that for $n \geq 3$ and $\delta(G) \geq n / 2$, we have $\operatorname{diam}(G) \leq 2$.

\section{Concluding remarks}

The quest for graphs other than $C_{11}$ which are solutions of Šoltes's problem has been completely unsuccessful so far. However, by studying a relaxed version of the problem or by focusing on some particular classes of graph, one could get a better insight into the original problem and find one more solution of it, or show that such graphs do not exist. 
One can consider regular graphs. Note that asking for a graph to be vertextransitive will be a as well a solution of the Šoltés's problem.

We can pose the following problems.

Problem 10. Are there $k$-regular connected graphs $G$ other than $C_{11}$ for which the equality $W(G)=W(G-v)$ holds for at least one vertex $v \in V(G)$ ?

We know that there are no such graphs for $k \geq n / 2$.

One can go further and study graphs $G$ for which equation $W(G)=W(G-S)$ holds for a subset $S$ of the vertex set $V(G)$ consisting of at least 2 vertices.

Problem 11. Find connected graphs $G$ for which

$$
W(G)=W(G-S)
$$

for any $S \subset V(G)$, with $|S| \geq 2$.

Our results show the existence of an infinite class of graphs $G$ for which $W(G)=$ $W(G-v)$ for a particular vertex $v$. It is natural to formulate the following conjecture.

Problem 12. For a given $r$, find (infinitely many) graphs $G$ for which

$$
W(G)=W\left(G-v_{1}\right)=W\left(G-v_{2}\right)=\cdots=W\left(G-v_{r}\right)
$$

for any distinct vertices $v_{1}, \ldots, v_{r} \in V(G)$.

Acknowledgements. The authors acknowledge partial support by Slovak research grants VEGA 1/0026/16, VEGA 1/0142/17 and APVV-15-0220, National Scholarschip Programme of the Slovak Republic SAIA and Slovenian research agency ARRS, program no. P1-0383.

\section{References}

[1] R. Diestel, Graph Theory: Electronic Edition 2000 Springer Verlag - New York (1997, 2000).

[2] A. A. Dobrynin, R. Entringer and I. Gutman, Wiener index of trees: theory and application, Acta Appl. Math. 66 (2001) 211-249.

[3] R. C. Entringer, D. E. Jackson and D. A. Snyder, Distance in graphs, Czechoslovak Math. J. 26 (1976) 283-296. 
[4] E. Estrada, The Structure of Complex Networks: Theory and Applications, Oxford University Press (2011).

[5] M. Knor, R. Škrekovski and A. Tepeh, Mathematical aspects on Wiener index, Ars Math. Contemp. 11 (2016) 327-352.

[6] M. Knor, S. Majstorović, R. Škrekovski, Graphs whose Wiener index does not change when a specific vertex is deleted, Discrete Appl. Math. 238 (2018) 126132 .

[7] M. Knor, S. Majstorović, R. Škrekovski, Graphs preserving Wiener index upon vertex removal, Appl. Math. Comput. 338 (2018) 25-32.

[8] O. E. Polansky, D. Bonchev, The Wiener number of graphs. I. General theory and changes due to some graph operations, MATCH Commun. Math. Comput. Chem. 21 (1986) 133-186.

[9] L'. Šoltés, Transmission in graphs: A bound and vertex removing, Math. Slovaca 41 (1991) 11-16.

[10] H. Wiener, Structural determination of paraffin boiling points, J. Am. Chem. Soc. 69 (1947) 17-20.

[11] K. Xu, M. Liu, K. C. Das, I. Gutman and B. Furtula, A survey on graphs extremal with respect to distance-based topological indices, MATCH Commun. Math. Comput. Chem. 71 (2014) 461-508. 